\title{
Lift of Slender Delta Wings According to Newtonian Theory
}

\author{
Arthur F. Messiter* \\ California Institule of Technology, Pasadena, Calif.
}

\begin{abstract}
An approximate system of equations is derived to describe the inviseid flow past a flat slender wing at angle of attack, in the limit $\gamma \rightarrow 1$ and $M_{\infty} \sin \alpha \rightarrow \infty$. The aspect ratio is required to approach zero at the same rate as the Mach angle in the flow behind the shock wave. Only a single parameter appears in the resulting equations, and a similarity law therefore can be written expressing a correction to the Newtonian normal-force coefficient. For the delta wing, a correlation of experimental data according to the similarity law is shown, and the first terms of the solution are derived under the assumption that the similarity parameter is small (vertex angle much smaller than Mach angle).
\end{abstract}

\section{Nomenclature}

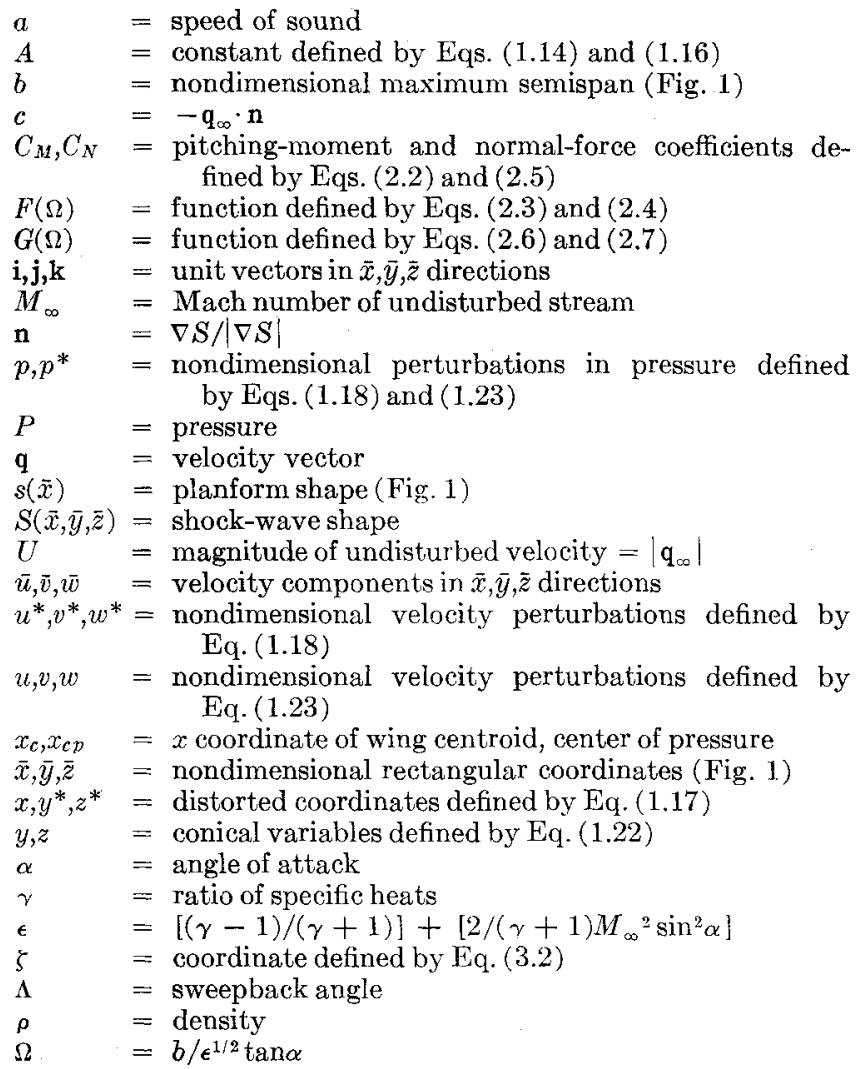

\section{Subscripts}

$b \quad=$ value at the wing surface

$s \quad=$ value immediately behind the shock wave

$\infty$

$=$ value in the undisturbed flow

\section{Introduction}

$\mathbf{T}$ HE flow past a body placed in a uniform stream is called hypersonic if two conditions are met: 1) the Mach number

Presented at the IAS National Summer Meeting, Los Angeles, Calif., June 19-22, 1962; revision received November 21, 1962. This work was supported partially by the Air Force Office of Scientific Research under Contract AF-49(638)476 and partially by the Hughes Aircraft Company. The author wishes to thank J. D. Cole for many helpful discussions.

* Senior Research Fellow; now Associate Professor of Aeronautical and Astronautical Engineering, University of Michigan, Ann Arbor, Mich. of the undisturbed flow is large compared to 1 ; and 2) changes in pressure and density occur which are of the same order as, or larger than, the undisturbed values. The Newtonian approximation used here involves the additional restrictions that the gas be perfect (this assumption is not essential) and inviscid, and that there occur very large changes in pressure and density. That is, the shock wave in front of the body is assumed to be "strong" in the sense that the Mach number normal to the shock is large and both the pressure and density of a fluid element increase greatly as the element erosses the shock. Over some portion of the body the shock will be very close to the surface. A detailed discussion of the approximation and its history is given by Hayes and Probstein. ${ }^{1}$

The Newtonian theory has been used in Refs. 2 and 3 in studying the hypersonic flow over lifting wings, subject to certain assumptions about the aspect ratio. In Ref. 2 it is implied that the aspect ratio is of order 1 in terms of the small parameters, and in Ref. 3 the aspect ratio is required to be extremely small. The related problem of conical flow over a body with sharp leading edges is discussed in Ref. 4, with the implicit assumption that the span and chord are of about the same size. For a lifting wing, an interesting intermediate case arises when the aspect ratio is of the same order of magnitude as the Mach angle in the flow behind the shock wave, i.e., when the effect of a flow disturbance is felt over a region having lateral extent of the same order as the wing span. If the wing has triangular planform, this case is expected to include two classes of flow patterns: one corresponding to a flow with the shock wave attached all along the leading edges, and the other corresponding to a shock attached only at the vertex.

The assumptions of Newtonian theory, together with the stated requirement of small aspect ratio, are used in the next section to derive an approximate analytical description of the hypersonic flow over a slender lifting wing. In the limit, the flow between the shock wave and the wing surface is identical with the flow behind a plane shock wave inclined to the stream at an angle equal to the wing angle of attack. The approximate equations describe the perturbations to this flow and, in principle, can be solved to give a correction to the pressure at the wing surface. On the expansion side of the wing, the pressure is somewhat smaller than $P_{\infty}$ and is taken equal to zero. A correction to the Newtonian normalforce coefficient $C_{N}=2 \sin ^{2} \alpha$ is expressed in terms of a function that depends on the single parameter $\Omega=b / \epsilon^{1 / 2} \tan \alpha$. The resulting similarity law (2.4), first reported in Ref. 5 , is used to correlate available experimental data for the lift of delta wings. These data give a tentative justification for the use of the theory.

For the delta wing, the general solution to the approximate 
differential equations is obtained easily by a transformation to characteristic coordinates. The boundary condition and shock relations then can be satisfied only if a complicated functional equation can be solved. A series expansion of the solution to this equation is assumed for $\Omega \rightarrow 0$, and the first two terms are derived. Integration then gives the initial terms of an expansion for $\Omega \rightarrow 0$ of the function $F(\Omega)$, which appears in the similarity law. This result is discussed in the last section.

\section{Approximation}

The coordinate system (Fig. 1) is chosen so that the wing, assumed flat, lies in the plane $\bar{y}=0$. The planform is described by $-b s(\bar{x})<\bar{z}<b s(\bar{x})$ and $0<\bar{x}<1$, where $s^{\prime}(\bar{x}) \geqslant$ 0 and $s(1)=1$. If the wing is at an angle of attack $\alpha$, as shown, a shock wave (not shown) exists below the wing. Provided that $\alpha$ is not close to $\pi / 2$, the shock is attached to the wing at the vertex and perhaps along part or all of the leading edges, depending on the geometry of the planform and on the values of the parameters. The region of interest is the high-pressure region bounded by the shock wave, the wing surface, and the surfaces $\bar{z}= \pm b s(\bar{x})$ and $\bar{x}=1$. It is typical of the Newtonian approximation that the pressure on the lower surface depends only on the flow field in this region. On the upper surface, the pressure will be neglected entirely.

For steady flow of an ideal gas, the continuity, momentum, and entropy equations may be written in the form

$$
\begin{gathered}
\operatorname{div} \rho \mathbf{q}=0 \\
\mathbf{q} \cdot \nabla \mathbf{q}+(1 / \rho) \nabla P=0 \\
\mathbf{q} \cdot \nabla\left(P / \rho^{\gamma}\right)=0
\end{gathered}
$$

In terms of rectangular coordinates $\bar{x}, \bar{y}, \bar{z}$ and corresponding velocity components $\bar{u}, \bar{v}, \bar{w}$, Eqs. (1.1) become

$$
\begin{gathered}
(\rho \bar{u})_{\bar{x}}+(\rho \bar{v})_{\bar{y}}+(\rho \bar{w})_{\bar{z}}=0 \\
\bar{u} \bar{u}_{\bar{x}}+\bar{v} \bar{u}_{\bar{y}}+\bar{w} \bar{u}_{\bar{z}}+(1 / \rho) P_{\bar{x}}=0 \\
\bar{u} \bar{v}_{\bar{x}}+\bar{v} \bar{v}_{\bar{y}}+\bar{w} \bar{v}_{\bar{z}}+(1 / \rho) P_{\bar{y}}=0 \\
\bar{u} \bar{w}_{\bar{x}}+\bar{v} \bar{w}_{\bar{y}}+\bar{w} \bar{w}_{\bar{z}}+(1 / \rho) P_{\bar{z}}=0 \\
\bar{u}\left[P_{\bar{x}}-(\gamma P / \rho) \rho_{\bar{x}}\right]+\bar{v}\left[P_{\bar{y}}-(\gamma P / \rho) \rho_{\bar{y}}\right]+ \\
\bar{w}\left[P_{\bar{z}}-(\gamma P / \rho) \rho_{\bar{z}}\right]=0
\end{gathered}
$$

At the surface $B(\bar{x}, \bar{y}, \bar{z})=0$ of any solid body, it is required that the normal component of velocity vanish; that is, $q \cdot \nabla B$ $=0$, where $q$ is evaluated at $B=0$. For the wing as shown in Fig. 1, this relation becomes

$$
\bar{v}(\bar{x}, 0, \bar{z})=0
$$

for $-b s(\bar{x})<\bar{z}<b s(\bar{x}), 0<\bar{x}<1$.

The solution in the high-pressure region is joined to the uniform flow upstream according to a set of jump conditions imposed at the shock wave:

$$
\begin{gathered}
{[\rho(\mathbf{q} \cdot \mathbf{n})]=0} \\
{\left[P+\rho(\mathbf{q} \cdot \mathbf{n})^{2}\right]=0} \\
{\left[\frac{1}{2}(\mathbf{q} \cdot \mathbf{n})^{2}+\{\gamma /(\gamma-1)\} P / \rho\right]=0} \\
{[\mathbf{q} \times \mathbf{n}]=0}
\end{gathered}
$$

where the square brackets denote the change in the enclosed quantity across the shock. The vector $\mathrm{n}$ is the unit vector normal to the shock wave, taken to be positive when directed away from the wing (i.e., "outward"). The first three of Eqs. (1.4) are scalar equations and are the usual jump conditions for mass, momentum, and stagnation enthalpy across a normal shock; the last is a vector equation expressing continuity of the tangential velocity.

If the shock shape is defined by

$$
S(\bar{x}, \bar{y}, \bar{z})=\mathbf{0}=\bar{y}-\bar{y}_{s}(\bar{x}, \bar{z})
$$

then the normal is

$$
\mathrm{n}=\frac{\nabla S}{|\nabla \bar{S}|}=\frac{-\bar{y}_{s_{\bar{x}}} \mathbf{i}+\mathbf{j}-\bar{y}_{s_{\bar{z}}} \mathbf{k}}{\left(1+\bar{y}_{\delta_{\tilde{x}}}{ }^{2}+\bar{y}_{s_{\bar{z}}}{ }^{2}\right)^{1 / 2}}
$$

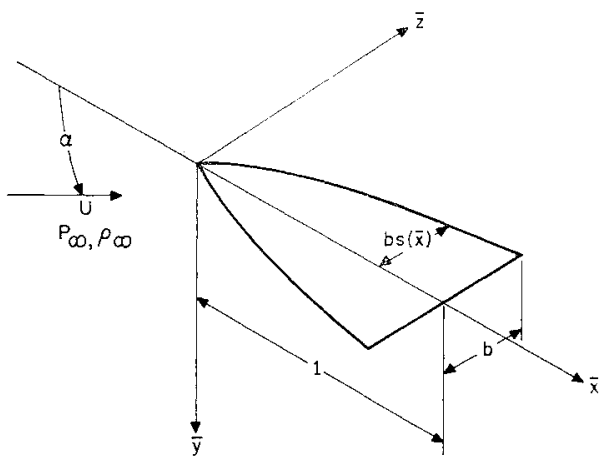

Fig. 1 Wing geometry

where $\mathbf{i}, \mathbf{j}, \mathbf{k}$ are unit vectors in the $\bar{x}, \bar{y}, \bar{z}$ directions. Let the subscripts $\infty$ and $s$ denote, respectively, the uniform-flow conditions upstream of the shock wave and the conditions immediately downstream of the shock. The speed at which the shock wave moves into the undisturbed fluid is $c=-q_{\infty} \cdot n$, where the minus sign appears because of the choice of direction for $\mathrm{n}$. The first three of Eqs. (1.4) can be solved for $\mathrm{q}_{s} \cdot \mathrm{n}, P_{s}$, and $\rho_{s}$ in terms of $c$ :

$$
\begin{aligned}
1-\frac{\rho_{\infty}}{\rho_{s}}=1+\frac{\mathrm{q}_{s} \cdot \mathrm{n}}{c}=\frac{2}{\gamma+1}\left(1-\frac{a_{\infty}^{2}}{c^{2}}\right) & = \\
& \frac{P_{s}-P_{\infty}}{\rho_{\infty} c^{2}}
\end{aligned}
$$

where $\mathbf{q}_{s}=\mathbf{q}\left(\bar{x}, \bar{y}_{s}, \bar{z}\right)=\bar{u}_{s} \mathbf{i}+\bar{v}_{s} \mathbf{j}+\bar{w}_{s} \mathbf{k}$, etc. The normal velocities $c$ and $\mathbf{q}_{s} \cdot \mathbf{n}$ are expressed in terms of the shock shape and $\bar{u}_{s}, \bar{s}_{s}, \bar{w}_{s}$ by

$$
\begin{aligned}
\frac{c}{U}= & \frac{\bar{y}_{s_{\bar{x}}} \cos \alpha+\sin \alpha}{\left(1+\bar{y}_{s_{\bar{x}}}{ }^{2}+\bar{y}_{\bar{s}_{\bar{z}}}{ }^{2}\right)^{1 / 2}} \\
\mathbf{q}_{s} \cdot \mathbf{n} & =\frac{-\bar{u}_{s} \bar{y}_{s_{\bar{x}}}+\bar{v}_{s}-\bar{w}_{s} \bar{y}_{s_{\bar{z}}}}{\left(1+\bar{y}_{s_{\bar{x}}}{ }^{2}+\bar{y}_{s_{\bar{z}}}{ }^{2}\right)^{1 / 2}}
\end{aligned}
$$

Resolution of the last of Eqs. (1.4) into $\bar{x}, \bar{y}, \bar{z}$ components gives

$$
\begin{aligned}
& 0=\left(-\bar{v}_{s} \bar{y}_{\bar{s}_{\bar{z}}}-\right.\left.\bar{w}_{\mathrm{s}}-U \bar{y}_{\bar{s}_{\bar{z}}} \sin \alpha\right) \mathbf{i}+ \\
&\left(\bar{u}_{s} \bar{y}_{s_{\bar{z}}}-\bar{w}_{s} \bar{y}_{s_{\bar{x}}}-U \bar{y}_{\bar{s}_{\bar{z}}} \cos \alpha\right) \mathbf{j}+ \\
& \quad\left(\bar{u}_{s}+\bar{v}_{s} \bar{y}_{s_{\bar{x}}}-U \cos \alpha+U \bar{y}_{s_{\bar{x}}} \sin \alpha\right) \mathbf{k}
\end{aligned}
$$

which is equivalent to two scalar equations, corresponding to two components of the tangential velocity. The system (1.6) plus (1.8) is thus sufficient to allow solution for $\bar{u}_{s}, \bar{v}_{s}$, $\bar{w}_{s}, P_{s}, \rho_{s}$ in terms of the (unknown) shock shape $\bar{y}_{s}$.

The Newtonian approximation implies that the shock wave must be close to the wing surface and that each of the quan-

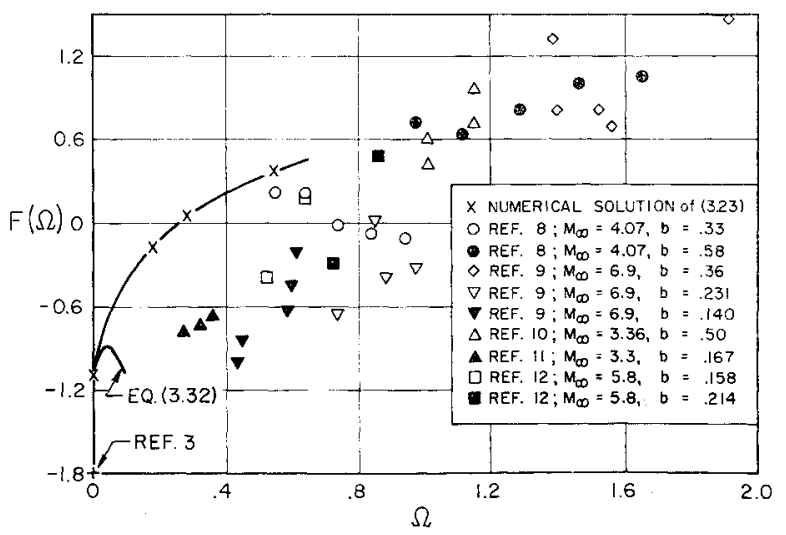

Fig. 2 Comparison of theory and experiment for delta wings, $\gamma=1.4$ 
tities $\bar{y}_{s_{\bar{x}}}, \bar{y}_{s_{\bar{z}}}$, and $\rho_{\infty} / \rho_{s}$ should be small compared to 1 . It follows from (1.6) and (1.7) that $(\gamma-1)$ and $\left(M_{\infty}^{2} \sin ^{2} \alpha\right)^{-1}$ are to be assumed small. That is, the approximation is expected to describe correctly the limit of the exact solution for $\gamma \rightarrow 1$ and $M_{\infty} \sin \alpha \rightarrow \infty$.

Since the shock wave lies close to the (flat) wing surface, the conditions immediately behind the shock are approximately the conditions behind a plane oblique shock inclined at an angle $\alpha$ to the uniform stream. Since the entropy of a fluid element remains constant after it has crossed the shock wave, and since the element undergoes only finite accelerations, the same conditions prevail throughout the thin layer between the shock wave and the wing surface. Hence one expects, throughout the region of interest,

$$
\begin{gathered}
\mathrm{q} / U \rightarrow \mathrm{i} \cos \alpha \\
P / \rho_{\infty} U^{2} \rightarrow \sin ^{2} \alpha \\
\epsilon\left(\rho / \rho_{\infty}\right) \rightarrow 1
\end{gathered}
$$

where $\epsilon$ is defined by

$$
\epsilon=[(\gamma-1) /(\gamma+1)]+\left[2 /(\gamma+1) M_{\infty}^{2} \sin ^{2} \alpha\right]
$$

One tries to find the first corrections to these limiting values by taking an appropriate limit of the differential equations and boundary conditions and then studying the resulting approximate system of equations. First the limit procedure must be stated carefully in terms of the nondimensional parameters $\gamma, M_{\infty}, \alpha$, and $b$. Then one can determine the behavior in the limit of $(\mathbf{q} / U)-\mathbf{i} \cos \alpha,\left(P / \rho_{\infty} U^{2}\right)-\sin ^{2} \alpha$, and $\left(\rho / \rho_{\infty}\right)-1$, each of which approaches zero in some manner. This second step amounts to determining the form of the first terms in the appropriate asymptotic expansions of these quantities.

As in various other solutions based on the Newtonian approximation, the limit procedure for $\gamma \rightarrow 1$ and $M_{\infty} \sin \alpha \rightarrow \infty$ will be defined such that

$$
(\gamma-1) M_{\infty}^{2} \sin ^{2} \alpha=\text { const }
$$

Uniform validity is expected for all but small values of this parameter; in particular, no fundamental change in the flow occurs if $M_{\infty}$ becomes arbitrarily large. It will be seen that no special simplification arises from a restriction to small deflections, and so also choose

$$
\alpha=\text { const }
$$

One should probably anticipate a nonuniformity for $\alpha \rightarrow \pi / 2$, since then the assumed limiting value of $q$ may no longer be larger than the perturbations.

The Mach angle in the flow behind the shock wave is approximately $\epsilon^{1 / 2} \tan \alpha$ and therefore tends to zero in the limit. For the delta wing, one expects that the region of nonuniform flow downstream of the vertex will have angular extent $O\left(\epsilon^{1 / 2}\right)$. If the aspect ratio is held fixed, then in the limit this region coincides with the plane $\bar{z}=0$, and discontinuities at $\bar{z}=0$ occur in some of the flow variables. ${ }^{2}$ On the other hand, if $b \rightarrow 0$ sufficiently fast (very slender wing), changes in the $\bar{x}$ direction become negligible, and the solution is given approximately by two-dimensional flow in transverse planes. $^{3}$ One might guess that the most interesting special case between these two is the case for which the aspect ratio tends to zero in such a manner that the vertex angle and the Mach angle are of the same order. Therefore let $b \rightarrow 0$ in such a manner that a parameter $\Omega$ remains fixed:

$$
\Omega=b / \epsilon^{1 / 2} \tan \alpha=\text { const }
$$

For a triangular planform, one might expect that the shock wave may be either attached or detached along the leading edges, depending on the value of $\Omega$.

A related solution that can serve as a useful guide is the approximate solution for high-speed flow past an infinitespan yawed wing at an angle of attack. ${ }^{6}$ If the parameters are such that the shock wave is attached along the leading edge, a uniform-flow solution can be found. The shock wave is plane, of the form

$$
\bar{y}_{s}(\bar{x}, \bar{z})=A(\bar{x}-\bar{z} \tan \Lambda)
$$

where $\Lambda$ is the sweepback angle ( $\tan \Lambda$ corresponds to $1 / b$ for the delta wing), and the constant $A$ may depend on $\gamma, M_{\infty}$, $\alpha$, and $\Lambda$. With the approximations $\bar{y}_{s_{\bar{x}}}, \bar{y}_{s_{\bar{Z}}}, \rho_{\infty} / \rho_{s} \ll 1$, the shock relations (1.6) and (1.8) become

$$
\begin{gathered}
\bar{u}_{s} / U=\cos \alpha-\bar{y}_{s_{\bar{x}}} \sin \alpha+\ldots \\
\bar{v}_{s} / U=\bar{y}_{s_{\bar{x}}} \cos \alpha-\bar{y}_{s_{\bar{z}}}{ }^{2} \sin \alpha-\epsilon \sin \alpha+\ldots \\
\bar{w}_{s} / U=-\ldots \\
\left(P_{s}-P_{\infty}\right) / \rho_{\infty} U^{2}=\sin ^{2} \alpha-\epsilon \sin ^{2} \alpha+2 \bar{y}_{s_{\bar{x}}} \sin \alpha \cos \alpha- \\
\bar{y}_{s_{\bar{z}}}{ }^{2} \sin ^{2} \alpha+\ldots \\
\rho_{\infty} / \rho_{s}=\epsilon+\left(1 / M_{\infty}{ }^{2} \sin ^{2} \alpha\right)\left\{-2 \bar{y}_{s_{\bar{x}}} \cot \alpha+\bar{y}_{s_{\bar{z}}}{ }^{2}\right\}+\ldots
\end{gathered}
$$

where the orders of magnitude of $\bar{y}_{s_{\tilde{x}}}=A$ and $\bar{y}_{s_{\bar{z}}}{ }^{2}=A^{2} \tan ^{2} \Lambda$ are yet to be determined. In the Newtonian limit, $\gamma \rightarrow 1$ and $M_{\infty} \sin \alpha \rightarrow \infty$, and the shock relation for $\bar{v}_{\varepsilon}$ becomes

$$
\bar{v}_{s} / U=0=A \cos \alpha-(A \tan \Lambda)^{2} \sin \alpha-\epsilon \sin \alpha+\ldots
$$

where $\bar{v}_{s}$ has been set equal to zero because the flow is uniform and satisfies the boundary condition (1.3). The solution for $A$ is

$$
\begin{aligned}
A / \epsilon \tan \alpha= & \left(1 / 2 \epsilon \tan ^{2} \alpha \tan ^{2} \Lambda\right) \times \\
& \left\{1 \pm\left(1-4 \epsilon \tan ^{2} \alpha \tan ^{2} \Lambda\right)^{1 / 2}\right\}+\ldots \text { (1.16a) }
\end{aligned}
$$

The result differs from that of Ref. 6 only because of the different coordinate system used. If $\tan \alpha$ and $\tan \Lambda$ are both held fixed as $\epsilon \rightarrow 0$, the lower sign leads to the ("weakshock") solution anticipated for two-dimensional flow:

$$
A=\epsilon \tan \alpha+\ldots
$$

If $\alpha$ is held fixed but $\Lambda$ increases, the square root becomes imaginary when $\tan \Lambda=O\left(\epsilon^{-1 / 2}\right)$; the vanishing of the square root is interpreted as a sufficient condition for shock detachment. Also, if $\tan \Lambda=O\left(\epsilon^{-1 / 2}\right)$, then $\bar{y}_{s_{\bar{x}}}$ and $\bar{y}_{s_{\bar{z}}}{ }^{2}$ are each $O(\epsilon)$, and it follows that $b=O\left(\epsilon^{1 / 2}\right)$ is the particular choice for the slender-wing problem which requires that all terms in (1.15) be retained. Thus the limit corresponding to $\Omega$ $=$ const represents a distinguished case mathematically as well as physically. Replacing $\tan \Lambda$ by $1 / b$ and rewriting the solution for $A$,

$$
A / \epsilon \tan \alpha=(\Omega / 2)\left[\Omega-\left(\Omega^{2}-4\right)^{1 / 2}\right]+\ldots
$$

The condition for detachment is $\Omega=2$, and the sign of the square root is chosen so that the solution approaches the two-dimensional value as $\Omega \rightarrow \infty$. Once $A$ is known, $\bar{y}_{s}(\tilde{x}, \bar{z})$ is known, and $\bar{u}, \bar{w}, P, \rho$ can be found from Eqs. (1.15).

The results for the swept wing will be used to suggest the asymptotic behavior of the flow over a slender wing. For a delta wing in the limit $\epsilon \rightarrow 0$, with $\alpha$ and $\Omega$ fixed, and $\Omega>2$, the yawed-wing solution might be expected to describe the flow in some neighborhood of the leading edge. Although one could make careful arguments about orders of magnitude, for a more general slender planform and for arbitrary $\Omega$, it is more convenient to assume that the orders will remain the same as found in the foregoing. Once a set of approximate equations has been obtained, one should try to show that these equations describe a mathematical problem for which a solution exists and is in some sense physically meaningful.

The (nondimensional) chord of the wing has been taken equal to 1 , the semispan $b$ is proportional to $\epsilon^{1 / 2} \tan \alpha$ (since $\Omega$ is held fixed), and the distance $\bar{y}_{s}$ to the shock wave varies as $\epsilon \tan \alpha$. The proper length scales for the $\bar{x}, \bar{y}$, and $\bar{z}$ directions therefore are taken to be $1, \epsilon \tan \alpha$, and $\epsilon^{1 / 2} \tan \alpha$, respectively. That is, if a point in the flow field between the shock wave and the wing surface is to keep the same relative position in the limit, the coordinates $\bar{y}$ and $\bar{z}$ should 
tend to zero in such a way that

$$
\begin{gathered}
x=\bar{x} \\
y^{*}=\bar{y} / \epsilon \tan \alpha \\
z^{*}=\bar{z} / \epsilon^{1 / 2} \tan \alpha
\end{gathered}
$$

are held fixed. The asymptotic representations therefore should be expressed in terms of these variables. The dependence on $\alpha$ shown in (1.17) and in the representations (1.18) below is not essential for deriving the approximate equations but is a convenience that leads to an especially useful form of these equations. Taking the yawed-wing solution as a guide, one expects that the following representations will describe the first corrections to the limiting values given by (1.9):

$$
\begin{gathered}
\bar{u}(\bar{x}, \bar{y}, \bar{z}) / U=\cos \alpha+\epsilon\left(\sin ^{2} \alpha / \cos \alpha\right) u^{*}\left(x, y^{*}, z^{*}\right)+\ldots \\
\bar{v}(\bar{x}, \bar{y}, \bar{z}) / U=\epsilon \sin \alpha v^{*}\left(x, y^{*}, z^{*}\right)+\ldots \\
\bar{w}(\bar{x}, \bar{y}, \bar{z}) / U=\epsilon^{1 / 2} \sin \alpha w^{*}\left(x, y^{*}, z^{*}\right)+\ldots
\end{gathered}
$$

$\left[P(\bar{x}, \bar{y}, \bar{z})-P_{\infty}\right] / \rho_{\infty} U^{2}=\sin ^{2} \alpha+\epsilon \sin ^{2} \alpha p^{*}\left(x, y^{*} z^{*}\right)+\ldots$

$$
\begin{aligned}
& \rho_{\infty} / \rho(\bar{x}, \bar{y}, \bar{z})=\epsilon-\epsilon^{2}\left[1+p^{*}\left(x, y^{*}, z^{*}\right)\right]- \\
& \quad[(\gamma-1) / 2] \epsilon\left[2 u^{*}\left(x, y^{*}, z^{*}\right)+w^{* 2}\left(x, y^{*}, z^{*}\right)\right]+\ldots
\end{aligned}
$$

$$
\bar{y}_{s}(\bar{x}, \bar{z})=\epsilon \tan \alpha y_{s}^{*}\left(x, z^{*}\right)+\ldots
$$

The correction to the density can be expressed in terms of the other perturbations, since one integral of the equations is known before any approximation is introduced: the stagnation enthalpy is constant everywhere. A nonuniformity for $\alpha \rightarrow \pi / 2$ is apparent in the representation for $\bar{u}$.

The basic assumption is that the representations (1.18), which show explicitly the dependence on the small parameter $\epsilon$, are uniformly valid throughout the region of interest between the wing surface and the shock wave. That is, one assumes, for example, that if $(\gamma-1) M_{\infty}^{2} \sin ^{2} \alpha, \alpha$, and $\Omega$ are held fixed, then

$$
\lim _{\epsilon \rightarrow 0}\left\{\frac{P(\bar{x}, \bar{y}, \bar{z})-P_{\infty}-\rho_{\infty} U^{2} \sin ^{2} \alpha}{\epsilon \rho_{\infty} U^{2} \sin ^{2} \alpha}\right\}
$$

exists uniformly for $0 \leqslant \bar{x} \leqslant 1,0 \leqslant \bar{y} \leqslant \bar{y}_{s}$, and $-b s(\bar{x}) \leqslant$ $\bar{z} \leqslant b s(\bar{x})$, except near the vertex of a pointed wing. The possibility of discontinuities may have to be investigated.

Since the variables on the left side of (1.18) depend on the four nondimensional parameters $\gamma, M_{\infty}, \alpha$, and $b$, and the dependence on $\epsilon$ is shown explicitly, it is implied that the starred variables defined by (1.18) may depend on three parameters that are held fixed as $\epsilon \rightarrow 0$, say, on $(\gamma-1) M_{\infty}{ }^{2} \sin ^{2} \alpha$, $\alpha$, and $\Omega$. But substitution of the assumed representations into the exact differential equations, shock-wave relations, and boundary condition shows that only the single parameter $\Omega$ remains in the approximate formulation of the problem. Taking the limit of the exact equations so as to retain only the lowest-order terms in $\epsilon$, one finds

$$
\begin{aligned}
& \frac{\partial v^{*}}{\partial y^{*}}+\frac{\partial w^{*}}{\partial z^{*}}=0 \\
& \frac{\partial u^{*}}{\partial x}+v^{*} \frac{\partial u^{*}}{\partial y^{*}}+w^{*} \frac{\partial u^{*}}{\partial z^{*}}=0 \\
& \frac{\partial v^{*}}{\partial x}+v^{*} \frac{\partial v^{*}}{\partial y^{*}}+w^{*} \frac{\partial v^{*}}{\partial z^{*}}=-\frac{\partial p^{*}}{\partial y^{*}} \\
& \frac{\partial w^{*}}{\partial x}+v^{*} \frac{\partial w^{*}}{\partial y^{*}}+w^{*} \frac{\partial w^{*}}{\partial z^{*}}=0
\end{aligned}
$$

$$
\begin{array}{rl}
u_{s}^{*} & =-\partial y_{s}^{*} / \partial x \\
v_{s}^{*} & =\left(\partial y_{s}^{*} / \partial x\right)-\left(\partial y_{s}^{*} / \partial z^{*}\right)^{2}-1 \\
w_{s}^{*} & =-\partial y_{s}^{*} / \partial z^{*} \\
p_{s}^{*} & =2\left(\partial y_{s}^{*} / \partial x\right)-\left(\partial y_{s}^{*} / \partial z^{*}\right)^{2}-1 \\
v^{*}\left(x, 0, z^{*}\right)=0 & 0<x<1 \quad-\Omega s(x)<z^{*}<\Omega s(x)
\end{array}
$$

where $p_{s}{ }^{*}=p\left(x, y_{s}{ }^{*}, z^{*}\right)$, etc. Since $\Omega$ is the only parameter appearing in these equations (in the boundary condition), the starred variables depend only on the coordinates $x, y^{*}, z^{*}$ and on $\Omega$. This result is used in the next section to derive certain similarity laws.

The equations show several features that are typical of the Newtonian approximation. For flows approximated in this manner, the pressure variation is related primarily to shockwave curvature and centrifugal forces, rather than to accelerations along a streamline. In the present case the curvature of the shock wave and of the streamlines is assumed vanishingly small because the wing is flat and the shock wave lies close to the wing surface; hence the pressure is nearly constant. Also, the magnitude of the velocity vector remains nearly constant along streamlines; that is, the momentum equations in the $x$ and $z^{*}$ directions are statements that the perturbations $u^{*}$ and $w^{*}$ are constant for each fluid element. The variation in pressure enters the differential equations only in relation to the small inclination of the velocity vector to the surface, i.e., in the momentum equation for the $y^{*}$ direction. The absence of a term $u_{x}{ }^{*}$ in the continuity equation is typical, since $|q|$ is nearly constant along streamlines, but the equation appears in the incompressible form only because the wing is flat and the density nearly constant.

The approximation also is typical in that the pressure can be found by direct integration, once the other variables are known. In principle, one first solves the continuity and $z^{*}$ momentum equations for $v^{*}$ and $w^{*}$. The equation for $u^{*}$ also could be solved, but the result is not needed unless one should want to calculate higher approximations; the equations for $u^{*}$ will be omitted in the following.

If the planform is triangular, then $s(x)=x$, and the system (1.19), (1.20), and (1.21) possesses conical symmetry. Define new variables

$$
\begin{aligned}
& y=y^{*} / x \\
& z=z^{*} / x
\end{aligned}
$$

The flow perturbations will be expressed by

$$
\begin{gathered}
v^{*}\left(x, y^{*}, z^{*} ; \Omega\right)=v(y, z ; \Omega) \\
w^{*}\left(x, y^{*}, z^{*} ; \Omega\right)=w(y, z ; \Omega) \\
p^{*}\left(x, y^{*}, z^{*} ; \Omega\right)=p(y, z ; \Omega)
\end{gathered}
$$

and the shock shape will be defined by

$$
y_{s}^{*}\left(x, z^{*} ; \Omega\right)=x y_{s}(z ; \Omega)
$$

Transformation of the differential equations gives

$$
\begin{aligned}
& v_{y}+w_{z}=0 \\
& (v-y) v_{y}+(w-z) v_{z}=-p_{y} \\
& (v-y) w_{y}+(w-z) w_{z}=0
\end{aligned}
$$

subject to the boundary condition

$$
v(0, z ; \Omega)=0
$$

for $-\Omega<z<\Omega$. The shock relations become

$$
\begin{aligned}
& v_{s}=y_{s}-z y_{s}{ }^{\prime}-y_{s}{ }^{2}-1 \\
& w_{s}=-y_{s}{ }^{\prime} \\
& p_{s}=2 y_{s}-2 z y_{s}{ }^{\prime}-y_{s}{ }^{2}-1
\end{aligned}
$$

Solution of the system (1.24), (1.25), and (1.26) is studied below, following the discussion of similarity laws. 


\section{Similarity Laws}

It was assumed that each of the flow variables has an asymptotic expansion uniformly valid in a certain region as $\epsilon \rightarrow 0$ with $(\gamma-1) M_{\infty}{ }^{2} \sin ^{2} \alpha, \alpha$, and $\Omega$ held constant. Since the approximate differential Eqs. (1.19), shock-wave relations (1.20), and boundary condition (1.21) involve only the parameter $\Omega$, these equations possess a similarity property that allows the number of parameters to be reduced to one. That is,

$$
p^{*}\left[x, y^{*}, z^{*} ;(\gamma-1) M_{\infty}^{2} \sin ^{2} \alpha, \alpha, \Omega\right]=p^{*}\left(x, y^{*}, z^{*} ; \Omega\right)
$$

etc.

Similarity laws can be written for each of the flow quantities and for integrated quantities such as the normal-force coefficient $C_{N}$. If the pressure on the upper surface of the wing is neglected, then

$$
\begin{aligned}
C_{N}= & \frac{\int_{0}^{1} d \bar{x} \int_{-b s(\bar{x})}^{b s(\bar{x})} P(\bar{x}, 0, \bar{z}) d \bar{z}}{\frac{1}{2} \rho_{\infty} U^{2} \int_{0}^{1} d \bar{x} \int_{-b s(\bar{x})}^{b s(\bar{x})} d \bar{z}} \\
= & 2 \sin ^{2} \alpha+\frac{2}{\gamma M_{\infty}^{2}}+ \\
& \epsilon \sin ^{2} \alpha \frac{2 \int_{0}^{1} d x \int_{-\Omega s(x)}^{\Omega s(x)} p^{*}\left(x, 0, z^{*} ; \Omega\right) d z^{*}}{\int_{0}^{1} d x \int_{-\Omega s(x)}^{\Omega s(x)} d z^{*}}+\ldots
\end{aligned}
$$

Since the ratio of integrals depends only on the shape of the planform and on $\Omega$, one may write (2.3) as an approximate similarity law for wings of geometrically similar planforms:

$$
\frac{C_{N}-2 \sin ^{2} \alpha-\left(2 / \gamma M_{\infty}^{2}\right)}{\epsilon \sin ^{2} \alpha}=F(\Omega)
$$

The rule (2.4) states that, if one knows the normal force on a slender wing flying at high Mach number for a particular set of values of $\gamma, M_{\infty}, \alpha$, and $b$, then one can calculate the force on a second wing that has a planform geometrically similar to that of the first, for a different set of values of $\gamma, M_{\infty}, \alpha$, and $b$, provided that the value of the parameter $\Omega$ remains unchanged. In other words, the calculation of $C_{N}\left(\gamma, M_{\infty}\right.$, $\alpha, b)$ for a family of wings having geometrically similar planforms, say for wings having a triangular planform, requires only the knowledge of a single function $F(\Omega)$.

A similarity law also can be derived for the pitching moment. In coefficient form, the moment about the $z$ axis is

$$
\begin{aligned}
C_{M} & =\frac{\int_{0}^{1} \bar{x} d \bar{x} \int_{-b s(\bar{x})}^{b s(\bar{x})} P(\bar{x}, 0, \bar{z}) d \bar{z}}{\frac{1}{2} \rho_{\infty} U^{2} \int_{0}^{1} d \bar{x} \int_{-b s(\bar{x})}^{b s(\bar{x})} d \bar{z}} \\
& =\left(2 \sin ^{2} \alpha+\frac{2}{\gamma M_{\infty}^{2}}\right) \frac{\int_{0}^{1} x d x \int_{-\Omega s(x)}^{\Omega s(x)} d z^{*}}{\int_{0}^{1} d x \int_{-\Omega s(x)}^{\Omega s(x)} d z^{*}} \\
+ & \epsilon \sin ^{2} \alpha \frac{2 \int_{0}^{1} x d x \int_{-\Omega s(x)}^{\Omega s(x)} p^{*}\left(x, 0, z^{*} ; \Omega\right) d z^{*}}{\int_{0}^{1} d x \int_{-\Omega s(x)}^{\Omega s(x)} d z^{*}}
\end{aligned}
$$

The similarity law is

$$
\frac{C_{M}-\left[2 \sin ^{2} \alpha+\left(2 / \gamma M_{\infty}^{2}\right)\right] x_{c}}{\epsilon \sin ^{2} \alpha}=G(\Omega)
$$

where $x_{c}$ is the $x$ coordinate of the centroid of the planform, and $G(\Omega)$ is defined by the ratio of integrals in the second term of (2.6). The distance from the wing vertex to the center of pressure is

$$
\begin{aligned}
x_{c p} & =C_{M} / C_{N} \\
& =\frac{\left[2 \sin ^{2} \alpha+\left(2 / \gamma M_{\infty}^{2}\right)\right] x_{c}+\epsilon \sin ^{2} \alpha G(\Omega)}{2 \sin ^{2} \alpha+\left(2 / \gamma M_{\infty}^{2}\right)+\epsilon \sin ^{2} \alpha F(\Omega)}
\end{aligned}
$$

Neglecting higher-order terms,

$$
x_{c p}-x_{c}=(\epsilon / 2)\left\{G(\Omega)-x_{c} F(\Omega)\right\}
$$

The planform for a delta wing is defined by $s(x)=x$, and the approximate flow field is described in terms of conical coordinates by the system of equations (1.24), (1.25), and (1.26). The pressure perturbation $p(0, z ; \Omega)$ at the wing surface is a function of a single variable, and the definition of $F(\Omega)$ becomes

$$
F(\Omega)=\frac{2}{\Omega} \int_{0}^{\Omega} p(0, z ; \Omega) a z
$$

Since the flow field is conical both in the exact theory and in the approximate theory,

$$
x_{c p}=x_{c}=\frac{2}{3}
$$

and, from (2.10),

$$
G(\Omega)=\frac{2}{3} F(\Omega)
$$

The first terms in an expansion of (2.11) for small values of $\Omega$ are found in the following section to be

$$
\begin{array}{r}
F(\Omega)=4(-1+\log 2)-8 \Omega \log \Omega+ \\
2 \Omega\left\{(3 \pi+6) \log 2-\left(\pi+10+\frac{\pi^{2}}{4}\right)-\right. \\
\left.4 \sum_{n=0}^{\infty} \frac{(-1)^{n}}{(2 n+1)^{\prime}}\right\}+\ldots
\end{array}
$$

It has not yet been possible to extend this result to larger values of $\Omega$.

A tentative verification of the theory is shown by a set of experimental results for delta wings correlated according to (2.4) and plotted in Fig. 2. The data have been selected from Refs. 8-12, subject to the requirements that $\epsilon$ and $b$ be small and that the change in local incidence due to wing thickness be negligible. The correlation is shown for values of the parameters in the ranges $0.2<\epsilon<0.4,0.1<b<0.6$, and $0.2<\Omega<2.0$. Other measurements reported in these references, but omitted from Fig. 2, either show the same trends as the data selected or else correspond to values of thickness ratio, of $\epsilon$, or of $b$ which seem obviously too large.

The points in Fig. 2 do appear to be grouped around a single curve, but the amount of scatter might at first seem rather large. However, the quantity plotted is the left-hand side of Eq. (2.4), which measures only a small part of the total normal force, and errors therefore appear considerably magnified. Error estimates given in the references, together with observed scatter in the data, suggest that the maximum error in the measured value of $C_{N}$ may be as large as 0.02 . If $M_{\infty} \sin \alpha=3.0$ and $\epsilon=0.26$, the corresponding error in $F(\Omega)$ is 0.66 for $\alpha=20^{\circ}$ and 0.15 for $\alpha=45^{\circ}$, significant fractions of the total scatter in Fig. 2. However, it is not clear that limitations in experimental accuracy can account for all of the scatter. It also is not obvious what values of $\epsilon$ and $b$ should be considered small. A conclusive demonstration of the accuracy and range of validity of the similarity law would require a set of tests conducted over wider ranges of the parameters than were chosen in the quoted references.

A numerical solution obtained from Eq. (3.23) also is shown in Fig. 2. The curve starts out somewhat above the experimental points and could be carried only as far as about $\Omega=$ 0.5 , because the accuracy of the procedure used appeared to become poorer for increasing $\Omega$. The analytical result for small $\Omega$ agrees exactly with the numerical result for $\Omega \rightarrow 0$, 
at $\Omega=0.005$, the difference is less than $0.5 \%$. But the first terms in the series obviously are not sufficient for values of $\Omega$ greater than about 0.02 . At $\Omega=0$ there is a discontinuity (discussed at the end of the next section) between these solutions and the solution of Ref. 3 .

At the other end of the curve, for $\Omega \rightarrow \infty$, one would guess that the measurements should approach the two-dimensional value $F(\Omega)=1$. As indicated in the references, the shock wave is detached from the leading edges for all of the points shown, and it is therefore not possible to check the behavior for $\Omega \rightarrow \infty$. Data for somewhat larger values of $b$ suggest that $F(\Omega)$ probably would increase to a maximum value greater than 1 and then decrease, perhaps sharply, to the limiting two-dimensional value.

Although additional theoretical and experimental work is needed, the results quoted are at least sufficient to indicate the possibility of useful semiempirical formulas for predicting $C_{N}$ for delta wings. The largest contribution to $C_{N}$ is the Newtonian term $2 \sin ^{2} \alpha$, and the data plotted in Fig. 1 therefore represent a relative correction approximately equal to $\frac{1}{2} \epsilon F(\Omega)$. For $M_{\infty} \sin \alpha=3.0, \epsilon=0.26$, and $|F(\Omega)| \leqslant 1$, the correction can be as large as $13 \%$, and for an uncertainty in $F(\Omega)$ of \pm 0.4 , the error in prediction of total lift would be within about $5 \%$. It therefore might be useful even to make as crude an estimate as $F(\Omega)=-1+\Omega$ for $0 \leqslant \Omega \leqslant 2$, and $F(\Omega)=1$ for $\Omega \geqslant 2$. Given an accurate expression for $F(\Omega)$, one also might consider including three further empirical corrections. For $1<M_{\infty} \sin \alpha<2$, the largest error could be corrected by including a rough estimate of the pressure on the expansion surface of the wing as a function of $M_{\infty} \sin \alpha$. For larger aspect ratios, the data given in the references suggest that the similarity parameter should be redefined with $b$ replaced by $b /\left(1+b^{2}\right)^{1 / 2}$, so that $\Omega$ is the ratio of the sine of the vertex half-angle to (approximately) the sine of the Mach angle. Thickness effects might be included in an empirical way by assuming the validity of a superposition procedure whereby the Newtonian term $2 \sin ^{2} \alpha$ is replaced by an integral over the surface of $2 \sin ^{2} \theta$, where $\theta$ is the exact local angle of incidence. None of these possible modifications appear to have a sound theoretical basis, but each appears to provide a correction in the proper direction and therefore might find useful application in extending the range of validity.

The apparent accuracy of Newtonian theory in this application should not be unexpected, since the theory has been used successfully in similar problems of flows past flat-faced bodies, ${ }^{3.7}$ which also involve small perturbations from a uniform pressure field. But it should be emphasized that a more thorough verification would require a set of experimental measurements for high Mach number and low aspect ratio over a wide range of values of the parameter $\Omega$. In addition to checking values of $C_{N}$, it would be desirable to correlate quantities related to the details of the flow fieldfor example, shock-wave shape and pressure distribution over the surface.

\section{Solution for the Delta Wing}

The approximate equations describing the flow over a delta wing are given by (1.24), (1.25), and (1.26). By first transforming to a set of characteristic coordinates, one easily can find the general solution of the differential equations. If the shock relations and boundary condition also can be satisfied, one then can solve explicitly for the perturbations in velocity and pressure and also evaluate the function $F(\Omega)$ in $\mathrm{Eq}$. (2.4). It actually has been possible to find only the first few terms of an expansion of the solution for small $\Omega$.

Characteristic surfaces of the complete equations for inviscid three-dimensional flow are the Mach surfaces and slip surfaces, across which certain discontinuities may exist. The characteristics in the present approximation for conical flow are given by curves $\eta(y, z)=$ const which satisfy

$$
\left|\begin{array}{lcr}
\eta_{y} & \eta_{z} & 0 \\
D \eta & 0 & \eta_{y} \\
0 & D \eta & 0
\end{array}\right|=\mathbf{0}
$$

where $D \eta$ denotes the approximate expression for the derivative of $\eta$ along a streamline:

$$
D \eta \equiv(v-y) \eta_{y}+(w-z) \eta_{z}
$$

Simplification of the determinant gives the condition

$$
\eta_{y}^{2} D_{\eta}=0
$$

The vanishing of the second factor corresponds to a set of characteristics that are the projections (along rays through the wing vertex) of streamlines on a plane $x=$ const. These curves also are characteristics of the complete equations for conical flow. The second family of characteristics are the lines $z=$ const. Characteristics of a similar nature are found in Refs. 3 and 7 and do not seem to represent any simple limit of the characteristics of the complete equations. The significant new feature is the absence of an elliptic region in the flow field.

The differential equations will be rewritten in terms of characteristic coordinates $\zeta$ and $z$, where $\zeta$ is a coordinate identifying the projected streamlines and therefore must satisfy

$$
(v-y) \zeta_{y}+(w-z) \zeta_{z}=0
$$

The choice of coordinates is made specifie by the additional condition

$$
\zeta\left(y_{s}, z\right)=z
$$

For any streamline, $\zeta$ is the value of the spanwise coordinate at the intersection of the streamline with the shock wave. The shock wave therefore is represented by the straight line $z=\zeta$, but the curve, say $z=z_{b}(\zeta)$, representing the wing surface is unknown. The mapping $(y, z) \rightarrow(z, \zeta)$ is one-toone, except that the centerline $\zeta=z=0$ maps into the origin in the transformed plane.

The flow perturbations expressed as functions of the new variables will be denoted by the same symbols as before; that is, $p(y, z) \rightarrow p(\zeta, z)$, etc. Partial derivatives of $\zeta(y, z)$ and of the inverse $y(\zeta, z)$ are related according to

$$
\zeta_{u}=1 / y_{\zeta} \quad \zeta_{z}=-\left(y_{z} / y_{\zeta}\right)
$$

The differential Eqs. (1.24) therefore become

$$
\begin{gathered}
v_{\zeta}+w_{z} y_{\zeta}-w_{\zeta} y_{z}=0 \\
(w-z) v_{z} y_{\zeta}=-p_{\zeta} \\
w_{z}=0
\end{gathered}
$$

and from (3.2), the transformation $(y, z) \rightarrow(\zeta, z)$ is described by

$$
y_{z}=(v-y) /(w-z)
$$

If one interprets $p_{s}=p(\zeta, \zeta)=p(z, z)$, etc., the shock relations (1.26) remain unchanged. At the wing surface, two conditions must be imposed:

$$
\begin{aligned}
& v\left[\zeta, z_{b}(\zeta)\right]=0 \\
& y\left[\zeta, z_{b}(\zeta)\right]=0
\end{aligned}
$$

The general solution of Eqs. (3.4-3.7) now can be found (and also has been obtained for an arbitrary planform). First, Eq. (3.6) gives

$$
w(\zeta, z)=w(\zeta)
$$

where $w(\zeta)$ is to be determined later from the shock relations and boundary conditions. The result (3.10) says that the sidewash remains constant along the projected streamlines. Next (3.4) and (3.7) can be solved simultaneously. Differ- 
entiation of (3.7) with respect to $\zeta$ allows elimination of $v(\zeta, z)$, and one obtains a second-order equation for $y(\zeta, z)$ :

$$
[w(\zeta)-z] y_{\zeta z}=-y_{\zeta}
$$

The solution contains two more unknown functions and can be expressed in the form

$$
y(\zeta, z)=y_{s}(z)-\int_{\zeta}^{z}\left[w\left(\zeta_{1}\right)-z\right] f^{\prime}\left(\zeta_{1}\right) d \zeta_{1}
$$

since $z=\zeta$ at the shock wave. Then (3.7) gives

$$
\begin{aligned}
& v(\zeta, z)=y_{s}(z)-\int_{\zeta}^{z}\left[w\left(\zeta_{1}\right)-w(\zeta)\right] f^{\prime}\left(\zeta_{1}\right) d \zeta_{1}+ \\
& {[w(\zeta)-z] y_{\mathrm{s}}^{\prime}(z)-[w(\zeta)-z][w(z)-z] f^{\prime}(z) }
\end{aligned}
$$

and the pressure is found from (3.5) by direct integration.

The shock relations are sufficient to eliminate all but one of the unknown functions from the general solution. Substitution of (3.10) and (3.12) into (1.26) gives

$$
\begin{gathered}
w(\zeta)=-y_{s}^{\prime}(\zeta) \\
f^{\prime}(\zeta)=1 /[w(\zeta)-\zeta]^{2}
\end{gathered}
$$

The solution for $v(\zeta, z)$ simplifies to

$$
\begin{aligned}
v(\zeta, z)= & y(\zeta, z)+[w(\zeta)-z] \times \\
& \left\{\int_{\zeta}^{z} \frac{d \zeta_{1}}{\left[w\left(\zeta_{1}\right)-\zeta_{1}\right]^{2}}-w(z)-\frac{1}{w(z)-z}\right\}
\end{aligned}
$$

and the pressure can be expressed by

$$
\begin{aligned}
& p(\zeta, z)=-1+2 y_{s}(z)+2 z w(z)-w^{2}(z)+ \\
& \quad\left\{-1+\frac{1}{[w(z)-z]^{2}}\right\} w^{\prime}(z) \int_{\zeta}^{z} \frac{\left[w\left(\zeta_{1}\right)-z\right]^{3}}{\left[w\left(\zeta_{1}\right)-\zeta_{1}\right]^{2}} d \zeta_{1}
\end{aligned}
$$

At the wing surface $z=z_{b}(\zeta)$, the boundary conditions will be rewritten as follows:

$$
\begin{aligned}
\mathbf{0}= & (d / d \zeta) y\left(\zeta, z_{b}\right) \\
= & \frac{w(\zeta)-z_{b}(\zeta)}{[w(\zeta)-\zeta]^{2}}+z_{b}{ }^{\prime}(\zeta)\left\{\int_{\zeta}^{z b(\zeta)} \frac{d \zeta_{1}}{\left[w\left(\zeta_{1}\right)-\zeta_{1}\right]^{2}}-\right. \\
& \left.w\left[z_{b}(\zeta)\right]-\frac{1}{w\left[z_{b}(\zeta)\right]-z_{b}(\zeta)}\right\}
\end{aligned}
$$

and

$$
\begin{aligned}
& 0=v\left(\zeta, z_{b}\right)-y\left(\zeta, z_{b}\right) \\
&=\left[w(\zeta)-z_{b}(\zeta)\right]\left\{\int_{\zeta}^{z b(\zeta)} \frac{d \zeta_{1}}{\left[w\left(\zeta_{1}\right)-\zeta_{1}\right]^{2}}-w\left[z_{b}(\zeta)\right]-\right. \\
&\left.\frac{1}{w\left[z_{b}(\zeta)\right]-z_{b}(\zeta)}\right\}
\end{aligned}
$$

By comparing (3.17) and (3.18), one finds two possibilities: either

$$
z_{b}(\zeta)=w(\zeta)
$$

or

$$
1 / z_{b}^{\prime}(\zeta)=0
$$

Equation (3.19a) states that a projected streamline $\zeta=$ const may terminate at a point on the wing surface where $z=w(\zeta)$. The alternative $(3.19 \mathrm{~b})$ says that $\zeta$ may remain constant over a portion of the surface.

If the shock wave is detached from the leading edge, the appropriate boundary condition is $(3.19 \mathrm{a})$, for $0 \leqslant z_{b}(\zeta) \leqslant \Omega$. The flow viewed in the $y, z$ plane resembles a two-dimensional flow in that plane, except that not all the fluid spills over the edges. A typical sketch of the curves $\zeta=$ const, for $0<\Omega<$ 2 , is shown in Fig. 3 . In the $\bar{x}, \bar{y}, \bar{z}$ coordinate system, the angle between a streamline and the $\bar{x}$ axis is approximately the ratio of velocities $\bar{w} / U \cos \alpha$. Since $w^{*}$ is constant for a fluid particle, each streamline is approximately a straight line and becomes relatively closer to a ray $\bar{z}=\bar{x} w(\zeta)$ as $\bar{x}$ increases. Hence the streamlines can be expected to terminate in the $y, z$ plane at a point where $z=w(\zeta)$.

If $\Omega$ is sufficiently large, the shock wave is attached, and a region of uniform flow might be expected in some neighborhood of the leading edge. A fluid element crossing the shock at the leading edge has $\zeta=\Omega$ and would remain on the surface as it moves away from the edge. The condition (3.19b) then would describe a uniform-flow region. The equations perhaps can be studied more easily for this case if the wing surface is defined by a curve $\zeta=\zeta_{b}(z)$ rather than $z=$ $z_{b}(\zeta)$. But a difficulty arjses in matching the uniform-flow solution to the nonuniform flow over the inboard region; the matching is not yet understood.

When the shock is detached, Eqs. (3.17) and (3.19a) can be combined to give a complicated functional equation for $w(\zeta)$

$$
w[w(\zeta)]+\frac{1}{w[w(\zeta)]-w(\zeta)}=\int_{\zeta}^{w(\zeta)} \frac{d \zeta_{1}}{\left[w\left(\zeta_{1}\right)-\zeta_{1}\right]^{2}}
$$

In this equation, $\zeta$ defines a (projected) streamline in terms of the spanwise coordinate at its intersection with the shock. Then $w(\zeta)$ is the sidewash on this streamline and, according to the boundary condition, also is the spanwise coordinate $z$ at the intersection of the streamline and the wing surface. The function $w[w(\zeta)]$, or $w(z)$, is the sidewash on another streamline, the one that intersects the shock at a point having spanwise coordinate $z$. Each of these variables is identified in Fig. 3.

Equation (3.20) is valid for $0 \leqslant w(\zeta)=z \leqslant \Omega$. Therefore, $\zeta$ varies between 0 and its value on the streamline which intersects the leading edge. Corresponding to the singularity in the transformation $(y, z) \rightarrow(\zeta, z)$, Eq. (3.20) has a singularity at $\zeta=w(\zeta)=0$. $A$ different sort of singularity occurs at $z=\Omega$. To show this, differentiate $(3.20)$ :

$w^{\prime}(\zeta) w^{\prime}[w(\zeta)]\left\{\frac{1}{\{w[w(\zeta)]-w(\zeta)\}^{2}}-1\right\}=\frac{1}{[w(\zeta)-\zeta]^{2}}$

The function $w^{\prime}[w(\zeta)]=w^{\prime}\left[z_{b}(\zeta)\right]$ represents the shock-wave curvature at the point $z=z_{b}$. When $w[w(\zeta)]-w(\zeta) \rightarrow 1$, $w^{\prime}[w(\zeta)] \rightarrow \infty$, and a singularity in shock-wave curvature occurs which resembles the singularity for two-dimensional flow past a flat plate normal to the stream ${ }^{3}$ or axisymmetric flow past a circular disk. ${ }^{7}$ As in these other problems, the singular point is expected to occur at the characteristic curve normal to the surface which passes through the edge, i.e., at $z=z_{b}(\zeta)=w(\zeta)=\Omega$. Hence the boundary conditions to. be included with (3.21) are

$$
\begin{gathered}
w(0)=0 \\
w(\Omega)=1+\Omega
\end{gathered}
$$

The condition (3.22a) is satisfied by a one-parameter family of solutions to (3.21); the desired solution is the one that also satisfies (3.22b).

Equations (3.21) and (3.22) represent one possible formulation of the problem that must be solved in order to find the flow field over a delta wing when the shock is detached. Although this form is the more easily derived and interpreted, a solution for $\Omega \rightarrow 0$ can be obtained more conveniently if one inverts the function $w(\zeta)$. The resulting formulation is

$$
\begin{aligned}
\frac{z^{\prime}(w) z^{\prime}[z(w)]}{\{z[z(w)]-z(w)\}^{2}} & =\frac{1}{[z(w)-w]^{2}}-1 \\
z(0) & =0
\end{aligned}
$$




$$
z(1+\Omega)=\Omega
$$

where $w(z)=w[w(\zeta)]$ has been replaced by $w, w(\zeta)$ by $z(w)$, and $\zeta$ by $z[z(w)]$. A Taylor's expansion about $w=1$ of the boundary condition $(3.23 \mathrm{c}$ ) gives a power series in $\Omega$. Assume that the solution also may be represented as a power series in $\Omega$ which is valid asymptotically for $\Omega \rightarrow 0$ :

$$
z(w)=\Omega z_{1}(w)+\Omega^{2} z_{2}(w)+\ldots
$$

Also assume that the shock wave $y_{s}(z)$ is analytic at $z=0$. It follows from symmetry that $z(w)$ is represented for small $w$ by a series of odd powers of $w$ and hence that

$$
\begin{aligned}
& z_{1}(w)=a_{1} w+a_{2} w^{3}+\ldots \\
& z_{2}(w)=b_{1} w+b_{2} w^{3}+\ldots
\end{aligned}
$$

Then

$$
\begin{aligned}
z[z(w)] & =z\left[\Omega z_{1}(w)\right]+\ldots \\
& =\Omega z_{1}\left[\Omega z_{1}(w)\right]+\ldots \\
& =a_{1} \Omega^{2} z_{1}(w)+\ldots
\end{aligned}
$$

Substituting the assumed expansions in (3.23),

$$
\begin{gathered}
a_{1} \Omega^{2} w^{2} z_{1}{ }^{\prime}(w)+\ldots= \\
z_{1}(1)+\ldots=1+\ldots
\end{gathered}
$$

For $\Omega \rightarrow 0$, the largest terms give

$$
\begin{aligned}
z_{1}(w) & =2 w /\left(1+w^{2}\right) \\
a_{1} & =2
\end{aligned}
$$

and the next terms can be shown to give

$$
\begin{aligned}
z_{2}(w) & =(\pi+4)\left[w /\left(1+w^{2}\right)\right]- \\
& \quad 8\left[w /\left(1+w^{2}\right)^{2}\right]\left(1+w \tan ^{-1} w\right) \\
b_{1} & =\pi-4
\end{aligned}
$$

The pressure $p_{b}$ at the wing surface is found by setting $z=z_{b}(\zeta)=w(\zeta)$ in (3.16) and then rewriting the result as a function of $w$ :

$$
\begin{aligned}
p_{b}= & -1+2 w z(w)-w^{2}+2 \int_{z(w)}^{w} \frac{w_{1}-z(w)}{\left[w_{1}-z\left(w_{1}\right)\right]^{2}} z^{\prime}\left(w_{1}\right) d w_{1}+ \\
& \left\{\frac{1}{[w-z(w)]^{2}}-1\right\} \frac{1}{z^{\prime}(w)} \int_{z(w)}^{w} \frac{\left[w_{1}-z(w)\right]^{3}}{\left[w_{1}-z\left(w_{1}\right)\right]^{2}} z^{\prime}\left(w_{1}\right) d w_{1}
\end{aligned}
$$

The first integral in (3.29) represents the shock-wave shape and is found by using the boundary condition (3.9) in the solution (3.11) for $y(\zeta, z)$. Expanding for small $\Omega$ as in the foregoing, one finds

$$
\begin{aligned}
& y_{s}=-2 \Omega \log 2 \Omega+\Omega\left\{\log \left(1+w^{2}\right)+\right. \\
& {\left.\left[2 /\left(1+w^{2}\right)\right]-4\right\}+\ldots . }
\end{aligned}
$$

The pressure becomes

$$
p_{b}=-\left[\left(1+w^{2}\right)^{2} / 2 w^{2}\right] \log \left(1+w^{2}\right)+\ldots
$$

Terms of order $\Omega \log \Omega$ and $\Omega$ also have been calculated. Then, from (2.11),

$$
\begin{aligned}
F(\Omega)= & \frac{2}{\Omega} \int_{0}^{1} p_{b} z^{\prime}(w) d w \\
= & 4(-1+\log 2)-8 \Omega \log \Omega \\
& +2 \Omega\left\{(3 \pi+6) \log 2-\left(\pi+10+\frac{\pi^{2}}{4}\right)-\right. \\
& \left.4 \sum_{n=0}^{\infty} \frac{(-1)^{n}}{(2 n+1)^{2}}\right\}+\ldots \\
& \approx-1.228-8 \Omega \log \Omega-17.17 \Omega+\ldots
\end{aligned}
$$

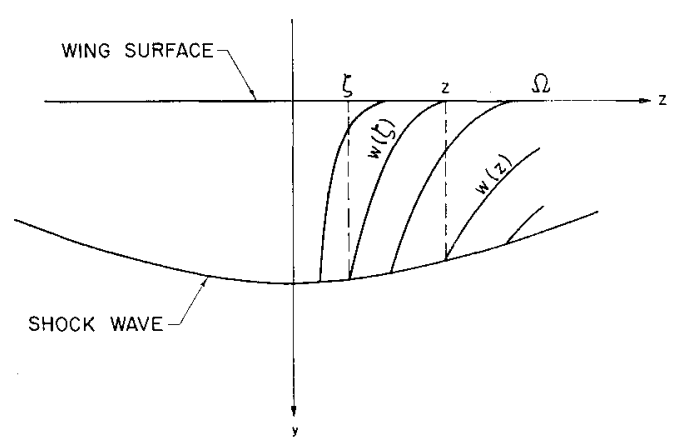

Fig. 3 Projected streamlines described by Eq. (3.20)

The result (3.32) agrees very well with a numerical solution of (3.23) in the limit $\Omega \rightarrow 0$ (see Fig. 2). Equation (3.32) is accurate only for extremely small $\Omega$ and so is useful primarily in helping to demonstrate the existence of a meaningful solution to the approximate equations. In Ref, 3 another solution was obtained, based on the Newtonian approximation and on the further assumption that changes in the chordwise direction could be neglected. One expects this solution to be correct in the limit $\epsilon \rightarrow 0$ if also $b \rightarrow 0$ sufficiently fast, and therefore if $\Omega \rightarrow 0$ sufficiently fast. The result for the integral of the surface pressure is expressed in the present notation by $F(0) \approx-1.80$ and does not agree with $\lim _{\Omega \rightarrow 0} F(\Omega)$ calculated from (3.32). It is believed that in the Newtonian approximation still another mathematical special case exists, corresponding to a limit such that $b / \epsilon$ is held fixed as $\epsilon \rightarrow 0$. Although this additional case may have little physical significance, it must be considered in order to complete the approximate mathematical description of the problem. In Ref. 3 it was found that very close to the surface the pressure gradient along the surface must appear in the approximate equations. For $b=O(\epsilon)$, the equations valid near the shock wave are the same as in Ref. 3 , but at the surface both the pressure gradient and changes in the chordwise direction must be taken into account. The derivation of Ref. 3 therefore assumes $b / \epsilon \rightarrow 0$. Although (3.32) and the result of Ref. 3 do not match each other, it is expected that each would match the solution corresponding to $b=O(\epsilon)$. $\dagger$

The theoretical results therefore are believed to be understood for small values of $\Omega$, and a tentative check with experiment has been obtained for values corresponding to a detached shock. It has not yet been possible to find a solution when the shock wave is attached. The difficulty, possibly related to the problem discussed in Ref. 4, arises in joining the solutions for a uniform flow (assumed near the leading edge) and a nonuniform flow. Also, it is not clear what sort of flow patterns will be predicted by the approximate theory when the shock wave detaches from the leading edges.

\section{References}

${ }^{1}$ Hayes, W. D. and Probstein, R. F., Hypersonic Flow Theory (Academic Press, New York, 1959).

${ }^{2}$ Gonor, A. L., "Hypersonic gas flow around conical bodies," Izv. Akad. Nauk SSSR, Otd. Tekhn. Nauk Mekhan. i Mashinostr., no. 1, 34-40 (1959); M. D. Friedman Transl. G-163.

${ }^{3}$ Cole, J. D. and Brainerd, J. J., "Slender wings at high angles of attack in hypersonic flow," ARS Preprint 1980-61 (1961).

${ }^{4}$ Melnik, R. E. and Scheuing, R. A., "Shock layer structure and entropy layers in hypersonic conical flows," ARS Preprint 1982-61 (1961); also Grumman Research Rept. RE-149 (1961).

$\dagger$ Kennet $^{13}$ uses the method of Belotserkovskii for a calculation of the hypersonic flow over a delta wing with $\Omega<2$. One of the results shown is a plot of $F(\Omega)$ vs $\Omega$, calculated by his method and compared with Fig. 2 of the present paper. His curve extends from $\Omega=0$ to $\Omega=0.8$, lies closer to the experimental data than does the present numerical solution, and agrees with the Cole and Brainerd result at $\Omega=0$. 
5 Messiter, A. F., "A similarity law for the normal force on a delta wing at hypersonic speeds," J. Aero/Space Sci. 26, 119-120 (1959).

${ }^{6}$ Cole, J. D., "Sweepback theory for shock waves at hypersonic speeds," Rand Rept. RM-1991 (1957).

7 Hida, K., "Blunt body theory for hypersonic flow," Air Force Office Sci. Research 204, Calif. Inst. Tech. (1961).

${ }^{8}$ Smith, F. M., "Experimental and theoretical aerodynamic characteristics of two low-aspect-ratio delta wings at angles of attack to $50^{\circ}$ at a Mach number of 4.07," NACA RM L57E02 (1957); declassified.

${ }^{9}$ Bertram, M. H. and McCauley, W. D., "An investigation of the aerodynamic characteristics of thin delta wings with a symmetrical double-wedge section at a Mach number of 6.9 ," NACA RM L55B14 (1955); declassified.

${ }^{10}$ Kaattari, G. E., "Pressure distributions on triangular and rectangular wings to high angles of attack-Mach numbers 2.46 and 3.36," NACA RM A54J12 (1955); declassified.

11 Hill, W. A., Jr., "Experimental lift of low-aspect-ratio triangular wings at large angles of attack and supersonic speeds," NACA RM A57117 (1957); declassified.

12 Nicholson, K. F., "The effects of blunt leading edges on delta wings at $M=5.8, "$ A. E. Thesis, Calif. Inst. Tech. (1958).

${ }^{13}$ Kennet, H., "The inviscid hypersonic flow on the windward side of a delta wing," Inst. Aerospace Sci. Paper 63-55 (January 1963).

\title{
Performance Evaluation of a Magnetically Spun d.c. Arc Operating in Nitrogen
}

\author{
D. R. Boldman* \\ NASA Lewis Research Center, Cleveland, Ohio
}

\begin{abstract}
The results of an experimental investigation of a concentric cylinder-type electrode configuration incorporating a magnetic field indicated that the are potential difference in nitrogen was essentially independent of the pressure in the range of 1 to $7 \mathrm{~atm}$ except for a specific range of operating conditions in which a $26 \%$ increase in arc potential difference was obtained. Highest efficiencies were achieved during the high potential operating mode. The independent effect of the electromagnetic force on the arc is not conclusive; however, experiments in which the magnetic field strength was varied over a moderate range indicated the importance of optimizing this effect from the standpoint of both chamber efficiency and arc stability. Photographs of the are reveal some of the effects of varying the magnetic field strength. The arc chamber efficiency was influenced also by the flow injection mode. An increase in efficiency was obtained when the flow opposed or was normal to the direction of the Lorentz force.
\end{abstract}

\section{Introduction}

A EROSPACE applications of the electric arc have introduced a number of new problem areas associated with the development of efficient, high-power arc chambers. One method, currently employed, to improve the performance of the high-power arc chamber consists of using electromagnetic forces to spin the are at very high angular speeds. Such forces can be either self-induced ${ }^{1}$ or applied by independent means as in Ref. 2 and the work to be described herein.

\section{Electrode Design and Limitations}

\section{Thoriated-Tungsten Cathode}

There are several advantages peculiar to this type of cathode. The arc becomes attached firmly to the pointed tip, as shown in Fig. 1, so that one end is fixed in space, thus providing good control of the longitudinal position of the are. Clean operation (contamination rates less than $0.1 \%$ by weight) for periods of several hours is possible if the cathode current limit is not exceeded.

A pointed tip has an advantage over the blunt tip from the standpoint of ease in starting; however, a blunt tip can conduct a higher current. The current limit of a 0.75 -in.-diam

Presented at the ARS Electric Propulsion Conference, Berkeley, Calif., March 14-16, 1962; revision received January 11, 1963.

* Aerospace Research Engineer. Member AIAA. pointed thoriated-tungsten cathode is approximately 1000 amp in nitrogen at pressures of 0.5 to $5 \mathrm{~atm}$. The current limit for a 0.75 -in.-diam flat-tipped cathode is about $1200 \mathrm{amp}$ under the same conditions; however, increasing the diameter of the flat tip to 1 in. permits operation at about $1500 \mathrm{amp}$ with little contamination. A 0.75 -in.-diam pointed tip can be employed in argon environments at current levels exceeding $5000 \mathrm{amp}$. Since the cathodes are current-limited, higher power levels can be achieved by either using multiple cathodes ${ }^{3},{ }^{4}$ or combining several units with a common plenum.

\section{Water-Cooled Copper Anode}

The anode consists of a water-cooled (coolant flow velocity of 16 fps at 60 psi gage) copper cylinder wound with a field coil (Fig. 1). Maximum magnetic flux densities of about 2.0 kgauss have been used. The anode has been subjected to several hours of operation at currents as high as $2100 \mathrm{amp}$ (using multiple cathodes) in nitrogen at a pressure of $2 \mathrm{~atm}$. In argon the anode has operated for several minutes at a current of $5200 \mathrm{amp}$ and a pressure of $0.5 \mathrm{~atm}$. The maximum power input in both nitrogen and argon environments is about $500 \mathrm{kw}$ for the forementioned coolant flow and magnetic flux density.

\section{Performance}

\section{Evaluation Procedure}

In the performance evaluation, four variables were controlled independently, namely: 1) the arc power input, 